\title{
ON THE DECOMPOSITIONS OF COMPLETE GRAPHS INTO CYCLES AND STARS ON THE SAME NUMBER OF EDGES
}

\author{
Atif A. Abueida And Chester Lian \\ Department of Mathematics \\ University of Dayton \\ 300 College Park, Dayton, OH 45469-2316 \\ e-mail: aabueida1@udayton.edu \\ lianc1@udayton.edu
}

\begin{abstract}
Let $C_{m}$ and $S_{m}$ denote a cycle and a star on $m$ edges, respectively. We investigate the decomposition of the complete graphs, $K_{n}$, into cycles and stars on the same number of edges. We give an algorithm that determines values of $n$, for a given value of $m$, where $K_{n}$ is $\left\{C_{m}, S_{m}\right\}$-decomposable. We show that the obvious necessary condition is sufficient for such decompositions to exist for different values of $m$.
\end{abstract}

Keywords: cycles, stars, graph-decompositions.

2010 Mathematics Subject Classification: 05C70.

\section{REFERENCES}

[1] A. Abueida, S. Clark and D. Leach, Multidecomposition of the complete graph into graph pairs of order 4 with various leaves, Ars Combin. 93 (2009) 403-407.

[2] A. Abueida and M. Daven, Multidesigns for graph-pairs of order 4 and 5, Graphs Combin. 19 (2003) 433-447.

doi:10.1007/s00373-003-0530-3

[3] A. Abueida and M. Daven, Multidecompositions of the complete graph, Ars Combin. 72 (2004) $17-22$.

[4] A. Abueida, M. Daven and K. Roblee, $\lambda$-fold multidesigns for graphs pairs on 4 and 5 vertices, Australas. J. Combin. 32 (2005) 125-136.

[5] A. Abueida and T. O'Neil, Multidecomposition of $\lambda K_{m}$ into small cycles and claws, Bull. Inst. Combin. Appl. 49 (2007) 32-40. 
[6] B. Alspach, Research problems, Problem 3, Discrete Math. 36 (1981) 333. doi:10.1016/S0012-365X(81)80029-5

[7] B. Alspach and H. Gavlas, Cycle decompositions of $K_{n}$ and $K_{n}-I$, J. Combin. Theory (B) 81 (2001) 77-99. doi:10.1006/jctb.2000.1996

[8] D. Bryant, D. Horsley, B. Maenhaut and B. Smith, Cycle decompositions of complete multigraphs J. Combin. Des. 19 (2011) 42-69. doi:10.1002/jcd.20263

[9] M. S̆ajna, Cycle decomposition III: complete graphs and fixed length cycles, J. Combin. Des. 10 (2002) 27-78. doi:10.1002/jcd.1027

[10] T. Shyu, Decomposition of complete graphs into paths and stars, Discrete Math. 310 (2010) 2164-2169. doi:10.1016/j.disc.2010.04.009

[11] T. Shyu, Decomposition of complete graphs into paths and cycles, Ars Combin. 97 (2010) 257-270.

[12] D. Sotteau, Decomposition of $K_{m, n}\left(K_{m, n}^{*}\right)$ into cycles (circuits) of length $2 k, \mathrm{~J}$. Combin. Theory (B) 30 (1981) 75-81. doi:10.1016/0095-8956(81)90093-9

[13] S. Yamamoto, H. Ikeda, S. Shige-eda, K. Ushio, and N. Hamada, On clawdecomposition of complete graphs and complete bigraphs, Hiroshima Math. J. 5 (1975) $33-42$.

Received 29 August 2012

Revised 22 December 2012

Accepted 22 December 2012 